\title{
Light Thoughts on Dark Energy
}

\author{
Eric V. Linder \\ Physics Division, Lawrence Berkeley National Laboratory, Berkeley, CA 94720
}

\begin{abstract}
The physical process leading to the acceleration of the expansion of the universe is unknown. It may involve new high energy physics or extensions to gravitation. Calling this generically dark energy, we examine the consistencies and relations between these two approaches, showing that an effective equation of state function $w(z)$ is broadly useful in describing the properties of the dark energy. A variety of cosmological observations can provide important information on the dynamics of dark energy and the future looks bright for constraining dark energy, though both the measurements and the interpretation will be challenging. We also discuss a more direct relation between the spacetime geometry and acceleration, via "geometric dark energy" from the Ricci scalar, and superacceleration or phantom energy where the fate of the universe may be more gentle than the Big Rip.
\end{abstract}

\section{Introduction}

The acceleration of the expansion of the universe poses a fundamental challenge to the standard models of both particle physics and cosmology. There is no established framework for the new physics required, but we know that within Einstein's field theory we can treat modifications to either the right hand side - energy-momentum components, e.g. a scalar field called quintessence - or the left hand side - the geometry of spacetime. Certainly the cosmological constant $\Lambda$ is equally at home in either location.

Here we examine what future observations can teach us about dark energy and the differences between a new physical component and an extension of gravitation theory. We give a very brief summary of dark energy theory, moving on to dark energy phenomenology - how to describe it in a manner amenable to both theory and observations, then dark energy fantasy - can we constrain complexities in the description, and finally dark energy reality - what we might expect to learn in the next decade. 


\section{Dark Energy Theory}

Guidance on the nature of dark energy from fundamental theory has been loose. While there is strong motivation for a cosmological constant, it encounters fine tuning and coincidence problems. Attempts to overcome these have led to tracer and tracker fields. The former, with a constant energy density relative to the dominant component, runs into problems with altered dynamics in the early universe, e.g. in the primordial nucleosynthesis era. The latter, where the field follows an attractor trajectory despite starting from a wide variety of initial conditions, has difficulties reaching an equation of state pressure to energy density ratio $w \equiv p / \rho<-0.7$, while current observations favor $w<-0.78$ at $95 \%$ confidence [Knop et al. 2003].

So theorists are currently in the state of trying anything and everything, including extra dimension models, tachyonic models, phase transition models, etc. With such a plethora of interest and examination, perhaps tomorrow a compelling model will leap forward from astro-ph or hep-th and convince the community that this is the natural, well motivated theory to work with. But it may be likelier that we will continue to have a surfeit of possibilities. The solution to this is likely to be survival of the fittest, in the original Darwinian sense: those theories that fit the data best will continue. This leads to the point of phenomenology - how to best interpolate between theory and observations so as to interpret the data robustly and cleanly.

\section{Dark Energy Phenomenology}

Observations to date have basically been of the expansion dynamics of the universe, one or another proxy for the scale factor as a function of time, $a(t)$. The Type Ia supernova method is the most direct tracer of this, with the redshift $z$ giving the scale factor and the luminosity giving the distance through the cosmological inverse square law, and hence the lookback time. To link to the underlying theory, but in a more model independent way, we can employ the equation of state (EOS) variable $w(z)$ as intermediary between $a(t)$ and, say, the scalar field potential $V(\phi)$. It is straightforward to carry out the translation between observations and a Lagrangian in theory, illustratively $V(\phi(a(t)))$.

As mentioned, observations already constrain the recent, averaged EOS to lie within about $15 \%(68 \% \mathrm{cl})$ of the value -1 . But the physics of the dark energy lies in its dynamics, the variation $w(z)$. Next generation experiments measuring distances and growth of structure should map $w(z)$ out to $z \approx 1.7$, if systematic effects from observational uncertainties and other astrophysical 
variations can be tightly controlled. One leading contender of a dark energy explorer is the Supernova/Acceleration Probe (SNAP: [snap.lbl.gov]), which is specifically designed for systematics control and employs both supernovae and weak gravitational lensing studies, as well as having further capabilities. Such a mission may constrain a measure of the time variation $w^{\prime} \equiv d w /\left.d \ln a\right|_{z=1}$ to within 0.08. The combination of high precision and high accuracy required certainly argues for a space mission.

Since the dynamics of the expansion is the key element linking the observations to the theory, we can see from the Friedmann equations for the expansion, involving both $\dot{a}$ and $\ddot{a}$, that we need to take into account not only the dark energy density but also its pressure. These can conveniently be combined in the equation of state ratio $w(z)$. However, this parametrization has greater applicability as well. A modification to the Friedmann equation can be translated to an effective parameter $w(z)$ :

$$
w(z) \equiv-1+\frac{1}{3} \frac{d \ln \left(\delta H^{2} / H_{0}^{2}\right)}{d \ln (1+z)},
$$

where $\delta H^{2}$ is defined to be $H^{2}-(8 \pi / 3) \rho_{m}$, i.e. whatever is not matter in the equation for the expansion rate $H=\dot{a} / a$.

This presents a wonderfully inclusive and model independent way to treat a large variety of dark energy theories, within the same "language". Of course, perhaps the resulting function $w(z)$ may look complicated, but this does not affect the mathematical treatment. And in fact, one finds that a remarkably simple but robust parametrization

$$
w(z)=w_{0}+w_{a}(1-a)=w_{0}+w_{a} z /(1+z),
$$

originally developed for slow roll scalar fields, works extremely well for many extensions to gravitation and high energy physics.

Figure 1 (left panel) illustrates this for an extra dimension braneworld model and a vacuum metamorphosis phase transition model. Note that these two theories span extremes in the sense of having averaged EOS near the upper and lower limits of current data, and possessing opposite signs of the time variation. Observations of both the growth history of structure in linear perturbation theory, written in terms of the gravitational potential $\Phi(z)$, and the expansion history in terms of the supernova magnitude-redshift relation (not shown, but fit to $0.01 \mathrm{mag}$ out to $z=2)$, can be superbly fit by $\left(w_{0}, w_{a}\right)$ with values of ($0.78,0.32)$ and $(-1,-3)$ respectively. This simplicity is a feature, not a bug, of the observations involving integrals over the EOS; that is, one does not attempt to reconstruct $w(z)$ (in particular in the case of vacuum metamorphosis this 
is not a good fit), but rather to match the observations going forward from the parametrization.
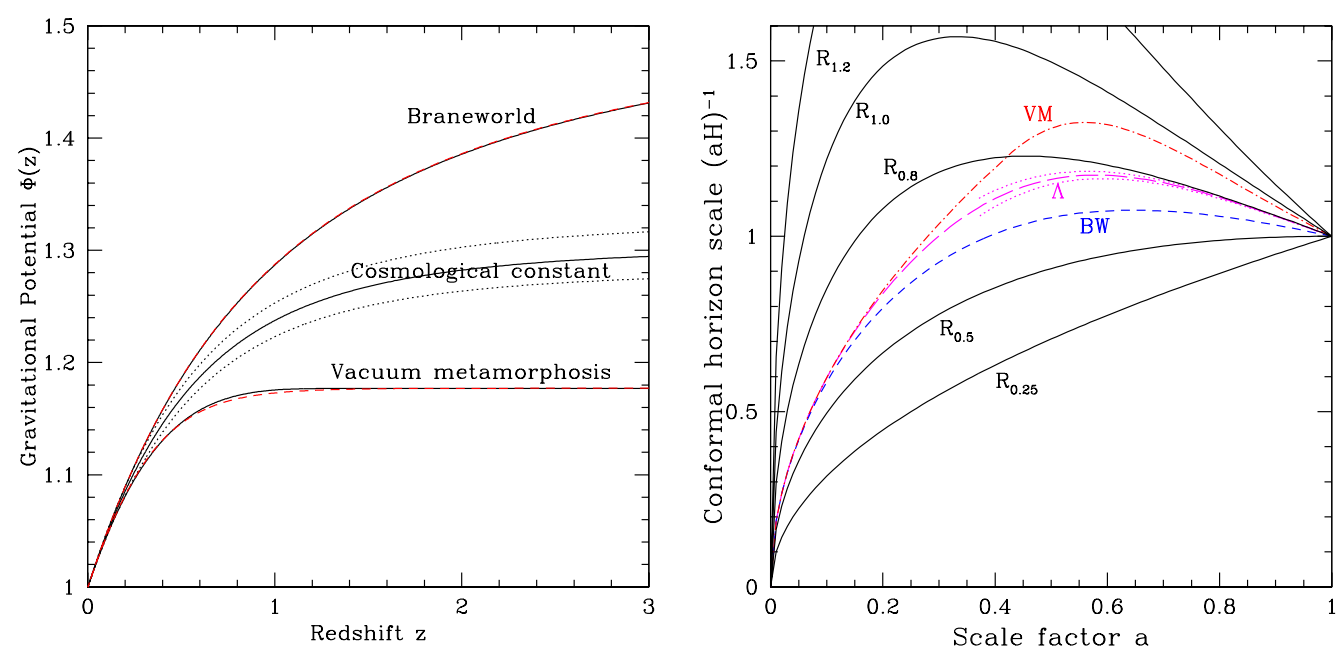

Fig. 1. [Left panel] The gravitational potential $\Phi(z)$ for two non-quintessence models shows the decay of the potential as the expansion accelerates. Dashed, red curves are for the mimicking quintessence models. Dotted outliers to the cosmological constant curve show the effect of changing the matter density $\Omega_{m}$ by 0.02 . [Right panel] The expansion history is plotted in terms of conformal horizon scale vs. scale factor for various modified gravity and spacetime geometry models. The Ricci geometric dark energy models (solid, black curves) are subscripted with the present value of $\mathcal{R}$. Negative slopes indicate an accelerating epoch while slopes more steeply negative than a critical value ( -1 at the present) indicate superacceleration.

Another possibility besides modifying the Friedmann equation is to attempt to address the acceleration directly through its relation to spacetime curvature, resident in the Principle of Equivalence. We can define a "geometric dark energy" based on the Ricci scalar curvature $R$, with $\mathcal{R}=R /\left(12 H^{2}\right)$ a key parameter in describing acceleration. This is related to parametrizations in terms of the second and third derivatives of the scale factor (e.g. "statefinder" from [Sahni et al. 2003] and "jerk" from [Visser 2003]) but gives a coherent foundation in terms of a geometric quantity rather than a Taylor expansion.

When $\mathcal{R}>1 / 2$ then the expansion of the universe accelerates and when $\mathcal{R}>1$ it superaccelerates. To make a close analogy with inflation theory acceleration in the early universe - consider the conformal diagram in the right panel of Figure 1. A negative slope to the conformal horizon scale $(a H)^{-1}$ with respect to $a$ means that comoving scales leave the horizon: just like in inflation a mark of acceleration. We see that this is achieved at the present epoch $(a=1)$ by the curve marked $R_{0.5}$, with $\mathcal{R}=1 / 2$ today. Superacceleration occurs for those models with curves steeper than $R_{1.0}$. We conjecture that enhanced particle production from the Rindler horizon created by superacceleration can obviate the Big Rip and create a cyclic scenario [Linder 2004]. 


\section{Dark Energy Fantasy}

If phenomenology describes the properties we expect the dark energy to possess, yet we must be aware of the possibility of more complicated situations where the basic characteristics of dark energy darkness, smoothness, and determination of the fate of the universe may not be so simple. Darkness can be lifted by self interactions or couplings to matter (which face stringent tests from fifth force laboratory experiments and astrophysical measurements of the matter power spectrum shape, e.g. [Sandvik et al. 2002]). Couplings to gravitation, e.g. in scalar-tensor theories of gravity, allow dark energy clumping on subhorizon scales, the possibility of backreaction from nonlinear structure formation, and maybe even a solution to the fine tuning problem through an attractor trajectory [Matarrese et al. 2004].

Without a complete theory of dark energy we cannot be sure of claims that measuring its density and time variation over a limited range of redshifts teaches us enough about dark energy to predict the fate of the universe. Even simple dark energy models, such as the linear potential, can have a currently accelerating phase that then gives way to a deceleration and collapse in a finite time [Kallosh et al. 2003].

\section{$5 \quad$ Dark Energy Reality}

The first step to learning about the new fundamental physics behind dark energy is measuring the time variation $w^{\prime}=w_{a} / 2$, with next generation experiments like SNAP. Before then, we may narrow in on a time averaged quantity $\langle w\rangle$, but this will mostly generate papers and speculation, not motivated theories. We have seen that even $w_{0}$ and $w_{a}$ go a long way toward fitting observations in terms of a real or effective equation of state function $w(z)$, and that $w(z)$ is an extremely general language for talking about the underlying physics.

Given this language, survival of the fittest enters, with the data deciding how or whether to go beyond a cosmological constant $\Lambda$. This will require careful and challenging levels of precision and systematics control, and needs to be complemented and crosschecked by multiple cosmological methods.

Part of the reality might be what lies in the future beyond $w(z)$. Consider the analogy with inflation. Inflation is not simply deSitter expansion: we want complexity - perturbations, running, tensor modes - to learn deeper physics. Similarly for dark energy we learn much through $w^{\prime}$ but there may detectable (some day) noncanonical sound speed $c_{s}^{2}$, inhomogeneities $\delta \phi$, couplings... 
Exciting times lie ahead for dark energy research.

\section{Acknowledgements}

This work has been supported in part by the Director, Office of Science, Department of Energy under grant DE-AC03-76SF00098. I thank David Cline for inviting me to present this overview of dark energy theory at the UCLA Dark Matter/Dark Energy 2004 meeting, and Robert Caldwell, Varun Sahni, Alexei Starobinsky, and Yun Wang for inspiring greater clarity of thought on various aspects.

\section{References}

[Knop et al. 2003] R. Knop et al. ApJ 598, 102 (2003) ; astro-ph/0309368

[snap.lbl.gov] SNAP: http://snap.lbl.gov

[Sahni et al. 2003] V. Sahni et al. JETP Lett. 77, 201 (2003) ; astro-ph/0201498

[Visser 2003] M. Visser, gr-qc/0309072

[Linder 2004] E.V. Linder, astro-ph/0402503

[Sandvik et al. 2002] H. Sandvik et al., astro-ph/0212114

[Matarrese et al. 2004] S. Matarrese, C. Baccigalupi, and F. Perrotta, astro-ph/0403480

[Kallosh et al. 2003] R. Kallosh et al. JCAP 310, 15 (2003) ; astro-ph/0307185 